\title{
Effect of tumor resection on the characteristics of functional brain networks
}

\author{
H. Wang, ${ }^{1}$ L. Douw, ${ }^{2}$ J. M. Hernández, ${ }^{1}$ J. C. Reijneveld, ${ }^{2}$ C. J. Stam,${ }^{3}$ and P. Van Mieghem ${ }^{1}$ \\ ${ }^{1}$ Faculty of Electrical Engineering, Mathematics, and Computer Science, Delft University of Technology, Delft, The Netherlands \\ ${ }^{2}$ Department of Neurology, VU University Medical Center, Amsterdam, The Netherlands \\ ${ }^{3}$ Department of Clinical Neurophysiology, VU University Medical Center, Amsterdam, The Netherlands \\ (Received 30 March 2010; revised manuscript received 5 July 2010; published 27 August 2010)
}

\begin{abstract}
Brain functioning such as cognitive performance depends on the functional interactions between brain areas, namely, the functional brain networks. The functional brain networks of a group of patients with brain tumors are measured before and after tumor resection. In this work, we perform a weighted network analysis to understand the effect of neurosurgery on the characteristics of functional brain networks. Statistically significant changes in network features have been discovered in the beta $(13-30 \mathrm{~Hz})$ band after neurosurgery: the link weight correlation around nodes and within triangles increases which implies improvement in local efficiency of information transfer and robustness; the clustering of high link weights in a subgraph becomes stronger, which enhances the global transport capability; and the decrease in the synchronization or virus spreading threshold, revealed by the increase in the largest eigenvalue of the adjacency matrix, which suggests again the improvement of information dissemination.
\end{abstract}

DOI: 10.1103/PhysRevE.82.021924

PACS number(s): 87.19.1j, 89.75.-k, 87.19.1e

\section{INTRODUCTION}

Complex networks are abstract expressions of the interactions or relations among elements within complex systems. Examples range from biological networks and manmade networks such as the Internet to social networks. The characteristics of networks have been extensively investigated to classify networks and to understand the effect of the network structure on its functioning [1-3].

The brain with its $10^{10}$ neurons and $10^{14}$ connections is one of the most challenging complex networks. Network theory has been applied to the human brain for almost a decade [4] and it has become clear that properties of brain networks may predict brain functioning such as cognitive performance $[5,6]$. Optimal brain functioning depends on functional interactions between brain areas [5,6], interconnected in the form of functional brain networks. The concept of functional connectivity refers to the statistical interdependencies between physiological time series recorded in various brain areas, and is thought to reflect communication between several brain areas [7]. Magnetoencephalography (MEG), a recording of the brain magnetic activity, is a method used to assess functional connectivity within the brain. A functional brain network is created by regarding each MEG channel as a node, and the functional connectivity between each pair of channels represents a link whose weight reflects the strength of the connectivity or correlation.

In this work, we compare functional brain networks of a group of patients with brain tumors before and after surgery, the aim of which was to remove the tumor. In brain tumor patients, it has been shown that brain networks are altered when compared to healthy controls $[8,9]$. Their network topologies seem more random, although contrary findings have been reported in aforementioned two studies. Thus, brain networks are known to be abnormal in brain tumor patients, but the effect-if any-of surgical treatment on these network abnormalities remains incompletely understood [10]. In this previous work, functional connectivity proved to change after the removal of the brain tumor. Especially in the theta band (4-8 Hz), a decrease in connectivity occurred, which was related to functional outcome in terms of epilepsy. As theta band connectivity has been reported to be pathologically increased in brain tumor patients $[8,9]$, this mechanism may represent a "normalization" after tumor resection. Our general goal in the current study is to identify changes in network characteristics when measured before and after tumor resection. In particular, we are interested to identify those topological characteristics of brain networks that might explain clinical changes in the postoperative situation.

Many real-world networks are represented as weighted networks using link weights to quantify a certain property of a link such as the distance or strength of a link. The characterization of weighted networks consists of studies (a) of the correlation between link weight structure and topology (see, e.g., [11-13]) and (b) of the link weight structure. In the second category (b), for example, the link weight correlation of links incident to a same node is examined in [14]. Since the functional brain networks are complete graphs, we investigate only the link weight structure via various link weight correlations. The second goal of this work is to propose weighted network analysis on the functional brain networks. In this paper, we introduce innovative measures to characterize weighted networks with respect to (a) link weight correlation of links incident to a node, (b) link weight correlation within triangles, and (c) clustering of high link weights in a subgraph. The spectrum, the eigenvalues of a weighted network, is also used to characterize the link weight correlation as well as the dynamic processes on a weighted network. These measures are shown to capture important changes in characteristics of functional brain networks measured before and after tumor resection.

Often, weighted functional brain networks are transformed into unweighted networks [5] by, e.g., mapping $L$ links with the highest link weight so that classical unweighted networks analysis can be applied afterward. We are going to illustrate that the weighted network analysis may infer, to some extent, properties in the so transformed un- 
TABLE I. Clinical characteristics of patients with brain tumors. $\mathrm{T}=$ temporal; $\mathrm{F}=$ frontal; $\mathrm{P}=$ parietal; $\mathrm{FT}=$ frontoparietal; $\mathrm{Me}=$ meningioma; $\mathrm{AI}-\mathrm{IV}=$ astrocytoma grade $\mathrm{I}-\mathrm{IV} ; \mathrm{OB}=$ oligodendroglioma grade $\mathrm{B}$; $\mathrm{OC}=$ oligodendroglioma grade $\mathrm{C} ; \mathrm{OD}=$ oligodendroglioma grade $\mathrm{D}$.

\begin{tabular}{|c|c|c|c|c|c|}
\hline Patient & Gender & Age & Lateralization & Localization & Histology \\
\hline 1 & M & 29 & $\mathrm{~L}$ & F & AIII \\
\hline 2 & F & 66 & $\mathrm{R}$ & $\mathrm{T}$ & $\mathrm{Me}$ \\
\hline 3 & F & 39 & $\mathrm{~L}$ & F & AIII \\
\hline 4 & M & 33 & $\mathrm{R}$ & $\mathrm{P}$ & AIII \\
\hline 5 & F & 30 & $\mathrm{R}$ & FP & AII \\
\hline 6 & M & 32 & $\mathrm{~L}$ & F & AII \\
\hline 7 & $\mathrm{~F}$ & 27 & $\mathrm{R}$ & FP & ОВ \\
\hline 8 & M & 31 & $\mathrm{~L}$ & F & AIV \\
\hline 9 & M & 38 & $\mathrm{~L}$ & F & AIII \\
\hline 10 & $\mathrm{~F}$ & 41 & $\mathrm{~L}$ & $\mathrm{P}$ & OC \\
\hline 11 & $\mathrm{~F}$ & 35 & $\mathrm{~L}$ & FT & AII \\
\hline 12 & M & 63 & $\mathrm{~L}$ & $\mathrm{P}$ & AIV \\
\hline 13 & $\mathrm{~F}$ & 48 & $\mathrm{R}$ & $\mathrm{T}$ & AII \\
\hline 14 & F & 26 & L & F & ОВ \\
\hline 15 & M & 43 & $\mathrm{R}$ & F & $\mathrm{Me}$ \\
\hline
\end{tabular}

weighted networks such as the degree variance, degree correlation, and clustering coefficient. The unweighted networks are shown to capture only partial characteristics of the original weighted network. These observations motivate a weighted network analysis as the most comprehensive strategy to explore the changes in network properties.

\section{CONSTRUCTION OF FUNCTIONAL BRAIN NETWORKS}

Neurons are electrically excitable cells in the nervous system that process and transmit information by electrochemical signaling. MEG is a noninvasive technique first used in 1968 to measure electric signals produced by neurons. Thus, MEG provides direct information about the dynamics of neural activity and the location of their sources in the brain. Sensorlevel MEG information has been used to assess brain networks on multiple occasions in past years, yielding accurate results [5,10,15-17]. Although MEG measurement always involves some methodological problems (e.g., volume conduction and inverse problem), these and other recent studies have shown that brain activity may indeed be measured using this type of recordings.

MEG measures brain activity with 151 recording channels, allowing for extensive analysis of functional connectivity between these different channels. Four artifact-free epochs of 4096 samples (6.5 s) were carefully selected by visual analysis from a data set of both the preoperative and postoperative registrations in brain tumor patients [10]. Subsequently, functional connectivity can be used as indication of link weight. In this case, the synchronization likelihood (SL) [18] was used as a measure of functional connectivity. SL is based on the concept of general synchronization [19] between two time series, which takes linear as well as nonlinear synchronization into account. The SL ranges between
$[0,1]$, with low SL indicating no synchronization and high SL meaning total synchronization. A network or graph can be deducted from the full matrix of SLs between all MEG channels. In such a graph, each channel represents a node and links exist between each node pair. The corresponding link weight is the SL between two nodes. One of the channels of the MEG apparatus was malfunctioning due to technical problems and could thus not be used for further analyses. Thus, the functional brain network we measured is a complete graph with $N=150$ nodes.

The functional brain networks are measured in 15 patients before and after brain tumor resection. The study protocol was approved by the medical ethics committee of the VU University Medical Center. All patients gave written informed consent before participating. Clinical characteristics of these patients can be seen in Table I. MEG was recorded during resting state, with eyes closed. From the total recording of $5 \mathrm{~min}$, four artifact-free epochs of 4096 samples (6.5 s) were selected; thus, the functional brain network is measured four times for each patient (see [10] for more information on selection methods). The calculations were carried out in five frequency bands: $0.5-4 \mathrm{~Hz}$ (delta), 4-8 Hz (theta), 8-10 Hz (lower alpha), 10-13 Hz (upper alpha), and 13-30 $\mathrm{Hz}$ (beta).

\section{WEIGHTED NETWORK ANALYSIS}

In this section, we apply our weighted graph analysis together with measures commonly employed in the literature to functional brain networks. The general goal is to identify the different characteristics of the brain networks measured before and after tumor resection. The relation among the weighted network measures or metrics will be also discussed. 


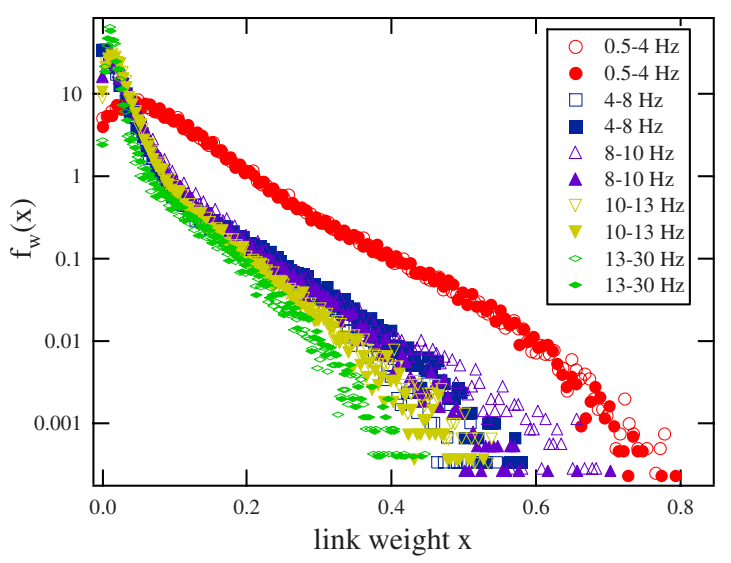

FIG. 1. (Color online) The pdf of a link weight in the brain functional networks measured before (empty markers) and after (solid markers) neurosurgery.

\section{A. Link weight and node strength distribution}

The probability density function (pdf) of link weights in functional brain networks measured before and after neurosurgery is given in Fig. 1. A similar link weight distribution (approximately exponentially distributed in the tail), as has been reported in anatomical and functional brain networks previously [20-22] is observed before and after neurosurgery in each frequency band. The average link weight is smaller for higher frequency bands. The link weight distribution is one of the most important features of weighted networks [23]. Many measures can be applied to compare different networks only if these networks possess the same link weight distribution, which will be further discussed in the later sections.

The node strength $s_{i}=\sum_{j \in \mathcal{N}(i)} w_{i j}$ is defined as the total weight of links connected to the node, where $\mathcal{N}(i)$ denotes the set of direct neighbors of node $i$. If the link weight $w_{i j}$ is considered as the strength of functional influence between nodes $i$ and $j$, the node strength characterizes the functional influence of a node over all the other nodes in a complete network. The node strength follows approximately a similar distribution $f_{s}(x)$ before and after neurosurgery, as shown in Fig. 2. However, after tumor resection, the maximal node strength reduces in frequency bands $0.5-4,8-10$, and 10-13 $\mathrm{Hz}$ and increases in 4-8 and 13-30 Hz. The changes in 4-8 $\mathrm{Hz}$ and 13-30 are statistically significant, implying that the global functional influence of hubs with respect to their node strengths is increased after neurosurgery. The increase in maximal node strength can be introduced by the increase in link weight correlation around a node, which is discussed at the end of Sec. III B.

\section{B. Link weight correlation around a node}

The link weight correlation of links incident to a node determines whether links connected to a same node tend to possess similar or dissimilar weights. We introduce three measures to examine the link weight correlation around a node.

\section{1. $\Sigma_{w}$}

A measure quantifying link weight correlation around a node has been proposed by Ramasco and Gonçalves [14]. It compares the ratio of the average link weight variance around each node to that of an ensemble of weight-reshuffled instances of the original graph. For example, the nonnormalized variance of the link weight around a node $i$ can be defined as
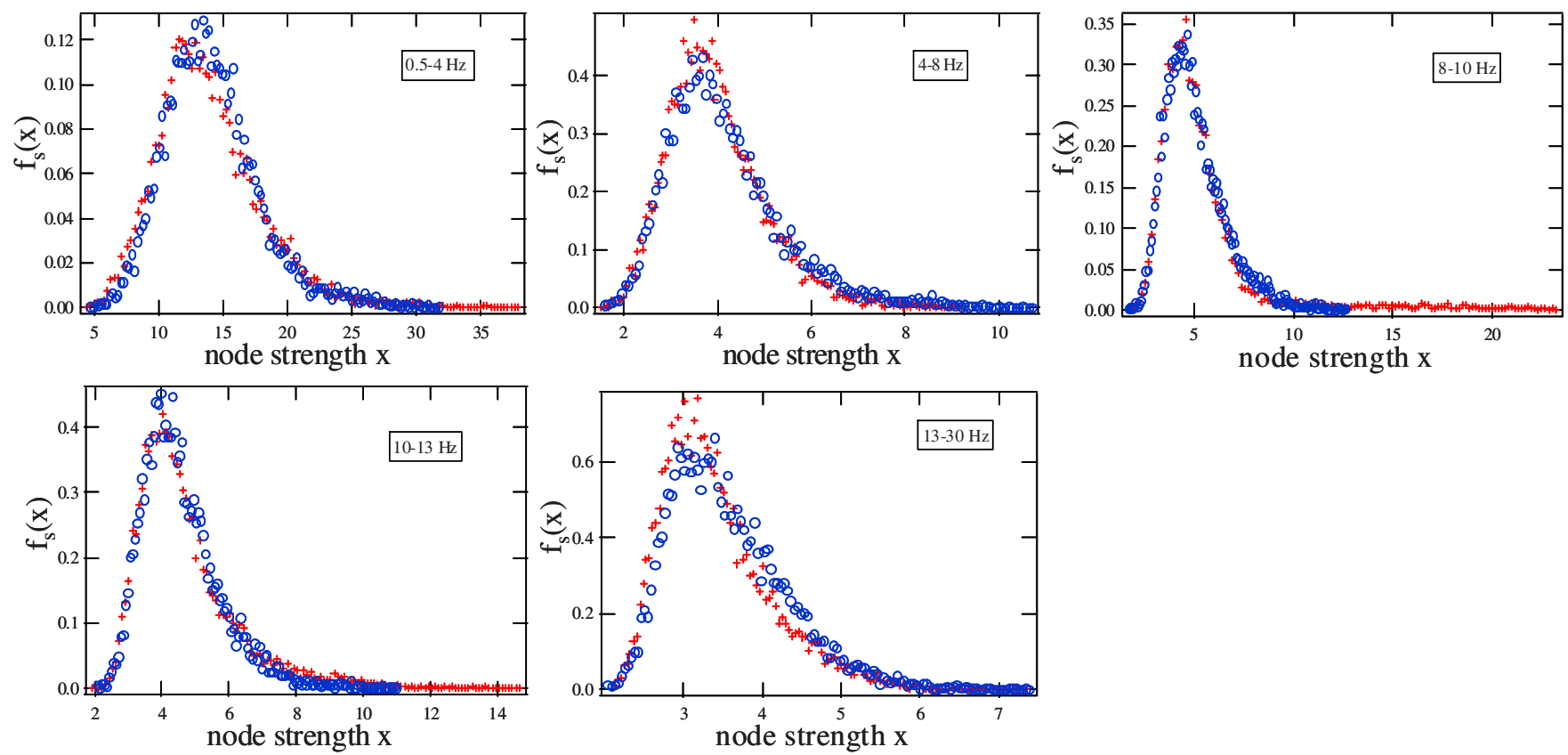

FIG. 2. (Color online) The pdf of node strength in MEG before (red crosses) and after (blue circles) neurosurgery. 
TABLE II. The link weight correlation $\Sigma_{w}(1)$ around a node in functional brain networks.

\begin{tabular}{cccc}
\hline \hline & $\begin{array}{c}E\left[\Sigma_{w}(G)\right] \text { before } \\
\text { neurosurgery }\end{array}$ & $\begin{array}{c}E\left[\Sigma_{w}\left(G^{\prime}\right)\right] \text { after } \\
\text { neurosurgery }\end{array}$ & $\begin{array}{c}\frac{E\left[\Sigma_{w}\left(G^{\prime}\right)\right]-E\left[\Sigma_{w}(G)\right]}{E\left[\Sigma_{w}(G)\right]} \\
(\%)\end{array}$ \\
\hline $0.5-4 \mathrm{~Hz}$ & 0.9456 & 0.9465 & 0.10 \\
$4-8 \mathrm{~Hz}$ & 0.9599 & 0.9502 & -1.01 \\
$8-10 \mathrm{~Hz}$ & 0.9418 & 0.9378 & -0.42 \\
$10-13 \mathrm{~Hz}$ & 0.9452 & 0.9442 & -0.11 \\
$13-30 \mathrm{~Hz}$ & 0.9654 & 0.9580 & -0.77 \\
\hline \hline
\end{tabular}

$$
\sigma_{w}^{2}(i)=\Sigma_{j \in \mathcal{N}(i)}\left(w_{i j}-\frac{\Sigma_{j \in \mathcal{N}(i)} w_{i j}}{d_{i}}\right)^{2},
$$

where $d_{i}$ is the degree of node $i$ and $\Sigma_{j \in \mathcal{N}(i)} w_{i j} / d_{i}$ is the average weight of the links arriving at $i$. The link weight correlation is then measured as

$$
\Sigma_{w}=\frac{E_{\text {org }}\left[\sigma_{w}\right]}{E_{\text {rand }}\left[\sigma_{w}\right]},
$$

where the average standard deviation is estimated for the original graph and an ensemble of weight-reshuffled [24] instances. The type of link weight correlation around each node in a network is revealed by comparing with the randomized instances: positive $\left(\Sigma_{w}<1\right)$, negative $\left(\Sigma_{w}>1\right)$, or noncorrelated $\left(\Sigma_{w}=1\right)$. The average link weight correlation $E\left[\Sigma_{w}\right]$ of functional brain networks derived based on (1) is shown in Table II.

\section{2. $\Sigma_{r}$}

The measure $\Sigma_{w}$ depends, however, on the link weight distribution. The link weight correlations around nodes in networks possessing different link weight distributions can be better compared via the rank correlation of links surrounding a node $\Sigma_{r}$. A link has rank $r$ if its link weight is the $r$ th largest among all the link weights in the network. If we replace each link weight $w_{i j}$ with the rank of that link, the rank correlation $\Sigma_{r}$ of links incident to a node is derived via Eq. (1). Tables II and III show that both measures point out the positive correlation since $\Sigma_{w}<1, \Sigma_{r}<1$; however, $\Sigma_{r}$ $\neq \Sigma_{w}$, because the rank of links in a network is uniformly distributed within $[1, L]$, where $L$ is the number of links in

TABLE III. The link weight correlation around a node $\Sigma_{r}$ of functional brain networks and its statistical tests.

\begin{tabular}{ccccc}
\hline \hline & $\begin{array}{c}E\left[\Sigma_{r}(G)\right] \\
\text { before } \\
\text { neurosurgery }\end{array}$ & $\begin{array}{c}E\left[\Sigma_{r}\left(G^{\prime}\right)\right] \\
\text { after } \\
\text { neurosurgery }\end{array}$ & $\begin{array}{c}\frac{E\left[\Sigma_{r}\left(G^{\prime}\right)\right]-E\left[\Sigma_{r}(G)\right]}{E\left[\Sigma_{r}(G)\right]} \\
(\%)\end{array}$ & $p$ \\
\hline $0.5-4 \mathrm{~Hz}$ & 0.9548 & 0.9557 & 0.094 & 0.933 \\
$4-8 \mathrm{~Hz}$ & 0.9807 & 0.9790 & -0.17 & 0.406 \\
$8-10 \mathrm{~Hz}$ & 0.9597 & 0.9656 & 0.61 & 0.756 \\
$10-13 \mathrm{~Hz}$ & 0.9678 & 0.9711 & 0.34 & 0.467 \\
$13-30 \mathrm{~Hz}$ & 0.9841 & 0.9804 & -0.38 & 0.062 \\
\hline \hline
\end{tabular}

the network, while the original link weights $w$ possess a distribution as shown in Fig. 1. Throughout this work, we study the link weight correlation around a node mainly via $\Sigma_{r}$ to take into account the small difference in link weight distribution before and after neurosurgery. As shown in Table III, link weights around a node are positively correlated in all functional brain networks since $\Sigma_{r}<1$. The correlation is stronger if $\Sigma_{r}$ is smaller. The most evident change is in $8-10$ Hz: $\left\{E\left[\Sigma_{r}\left(G^{\prime}\right)\right]-E\left[\Sigma_{r}(G)\right]\right\} / E\left[\Sigma_{r}(G)\right]=0.61 \%$. However, the evident change in the average link weight correlation $E\left[\Sigma_{r}\right]$ does not imply that this change occurs in each patient or in most patients.

In addition to visual inspection of the means in Table I, the statistical significance of these results is investigated. A statistically significant finding indicates that the results are unlikely to have occurred by chance, while a statistically nonsignificant finding is more likely due to chance. In this study, our hypothesis is that graph measures change after surgery in brain tumor patients, and the level of significance refers to the likeliness of this hypothesis not being rejected. In order to test the statistical significance of changes in a graph measure that was calculated both before and after tumor resection, nonparametric statistical tests are used. Most common statistical procedures, such as Student's t-tests and analysis of variance, assume a normal or Gaussian distribution of observed values. However, functional connectivity and graph theoretical measures usually have a skewed distribution, warranting nonparametric testing. The nonparametric Wilcoxon signed rank test compares two repeated measurements in the same sample by ranking both measurements [25]. With this nonparametric procedure, no assumptions regarding the underlying distribution of values are made. The changes in link weight correlation $\Sigma_{r}\left(G^{\prime}\right)-\Sigma_{r}(G)$ in each network are statistically tested using the Wilcoxon signed rank test, as shown in Table III. The $p$ value that is chosen to represent the level of significance of the observed results indicates whether the observed result is by chance more likely to deviate from the null hypothesis that there was no change after surgery. Thus, a $p$ value below 0.05 (which is the most commonly used threshold of significance $\alpha$ ) means that there is more than $95 \%$ chance that the null hypothesis can be rejected, and it implies that changes after surgery are actually present in the current study. There are no significant changes in the five frequency bands with respect to link weight correlation, although a trend toward significance was observed in the beta band. Other network measures to be examined may change significantly in different frequency bands. We always provide a table like Table III for each network measure in all frequency bands and also to understand the relation among all the measures.

$$
\text { 3. }\left(s_{i}+s_{j}\right) / 2(N-1) \text { versus } w_{i j}
$$

A third measure of link weight correlation around a node examines the relation between the weight $w_{i j}$ of a link $(i, j)$ and the normalized node strength $\left(s_{i}+s_{j}\right) / 2(N-1)$ of nodes $i$ and $j$. Since each node in the functional brain network is connected to all the other $N-1$ nodes, the average link weight incident to a node is $\frac{s}{N-1}$. The averaged $\left(s_{i}+s_{j}\right) / 2(N$ $-1)$ is actually the average link weight around nodes $i$ and $j$. 

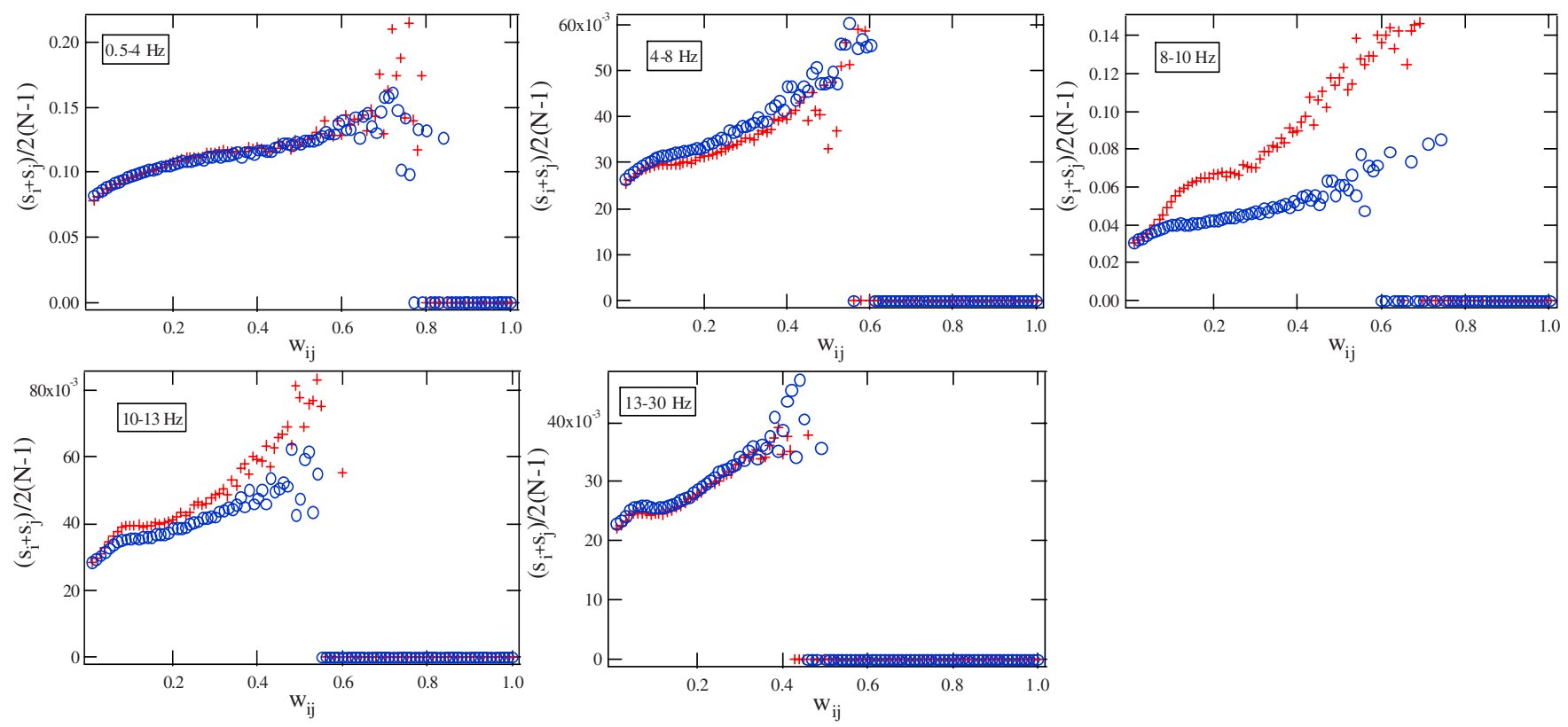

FIG. 3. (Color online) Correlation between $\left(s_{i}+s_{j}\right) / 2(N-1)$ and $w_{i j}$ of the brain function networks before (red crosses) and after (blue circles) neurosurgery.

The relation of $\left(s_{i}+s_{j}\right) / 2(N-1)$ versus $w_{i j}$ is examined over all the links in a network. If $\left(s_{i}+s_{j}\right) / 2(N-1)$ and $w_{i j}$ are positively correlated, a high link weight $w_{i j}$ implies a high average link weight around nodes $i$ and $j$. In this case, links connected to nodes $i$ and $j$ possess similar link weight as $w_{i j}$ [26]. A positive link weight correlation around a node can be expected.

In contrast to the geometric mean $\sqrt{s_{i} s_{j}}$, the arithmetic mean $\left(s_{i}+s_{j}\right) / 2(N-1)$ allows for theoretical analysis. An upper bound for the covariance of $\left(s_{i}+s_{j}\right) / 2$ and $w_{i j}$ can be deduced as follows:

$$
\begin{aligned}
\operatorname{Cov}\left[\frac{s_{i}+s_{j}}{2}, w_{i j}\right]= & E\left[\frac{s_{i}+s_{j}}{2} \cdot w_{i j}\right]-E\left[\frac{s_{i}+s_{j}}{2}\right] E\left[w_{i j}\right] \\
= & \frac{1}{2}\left(\sum_{k \in \mathcal{N}(i)} E\left[w_{i k} \cdot w_{i j}\right]+\sum_{q \in \mathcal{N}(j)} E\left[w_{j q} \cdot w_{i j}\right]\right) \\
& -(N-1)(E[w])^{2},
\end{aligned}
$$

where $E\left[s_{i}\right]=(N-1) E[w]$, because the functional brain networks are complete graphs. If all link weights surrounding a node are independent, then

$$
\begin{aligned}
\operatorname{Cov}\left[\frac{s_{i}+s_{j}}{2}, w_{i j}\right]= & \frac{1}{2}\left(\sum_{k \in \mathcal{N}(i)} E\left[w_{i k}\right] E\left[w_{i j}\right]\right. \\
& \left.+\sum_{q \in \mathcal{N}(j)} E\left[w_{j q}\right] E\left[w_{i j}\right]\right)-(N-1)(E[w])^{2} \\
= & 0 .
\end{aligned}
$$

When all link weights around a node are maximally correlated, $w_{i k} \cdot w_{i j}=w_{i j}^{2}$ and $E\left[w_{i k} \cdot w_{i j}\right]=E\left[w_{i j}^{2}\right]$. In this case,

$$
\begin{aligned}
\operatorname{Cov}\left[\frac{s_{i}+s_{j}}{2}, w_{i j}\right] & =(N-1) E\left[w^{2}\right]-(N-1)(E[w])^{2} \\
& =(N-1) \operatorname{Var}[w] .
\end{aligned}
$$

Therefore, the covariance of $\left(s_{i}+s_{j}\right) / 2$ and $w_{i j}$ is always upper bounded by $\operatorname{Cov}\left[\left(s_{i}+s_{j}\right) / 2, w_{i j}\right] \leq(N-1) \operatorname{Var}[w]$. The linear correlation coefficient between $\left(s_{i}+s_{j}\right) / 2$ and $w_{i j}$ or, approximately, $\operatorname{Cov}\left[\left(s_{i}+s_{j}\right) / 2, w_{i j}\right]$ illustrates how the scatter plots in Fig. 3 are close to a line. The slope of $\left(s_{i}+s_{j}\right) / 2(N$ -1) versus $w_{i j}$ reflects the relative strength of link weight correlation surrounding a node.

In Fig. 3, the relation between $\left(s_{i}+s_{j}\right) / 2(N-1)$ and $w_{i j}$ is illustrated [27]. The measure $E\left[\Sigma_{r}\right]$ in Table III and the measure $\left(s_{i}+s_{j}\right) / 2(N-1)$ and $w_{i j}$ in Fig. 3 reveal the same changes in link weight correlation around a node after tumor resection over all frequency bands: whenever $E\left[\Sigma_{r}\left(G^{\prime}\right)\right]$ $-E\left[\Sigma_{r}(G)\right]<0$, the slope of $\left(s_{i}+s_{j}\right) / 2(N-1)$ versus $w_{i j}$ increases. While the relation $\left(s_{i}+s_{j}\right) / 2(N-1)$ versus $w_{i j}$ provides the average link weight around each link $w_{i j}$ in a network, $\Sigma_{r}$ allows us to quantify the link weight correlation around nodes without curve fitting and thus can be applied to a large number of networks.

Another observation is that after neurosurgery the link weight correlation surrounding a node (see Table III) increases (decreases) when the maximal node strength (see Fig. 2) increases (decreases) in each frequency band: $\left\{E\left[\Sigma_{r}\left(G^{\prime}\right)\right]-E\left[\Sigma_{r}(G)\right]\right\}\left\{s_{\max }\left(G^{\prime}\right)-s_{\max }(G)\right\}<0$. This can be understood as follows: both high link weights and a strong positive link weight correlation around a node contribute to a high node strength. Since a similar link weight distribution is followed before and after neurosurgery in most bands, the maximal node strength is mainly determined by the link weight correlation. 
TABLE IV. The weighted clustering coefficient $C$ of functional brain networks measured before and after neurosurgery and its statistical tests.

\begin{tabular}{ccccc}
\hline \hline & $\begin{array}{c}E[C(G)] \text { before } \\
\text { surgery }\end{array}$ & $\begin{array}{c}E\left[C\left(G^{\prime}\right)\right] \text { after } \\
\text { surgery }\end{array}$ & $\begin{array}{c}\frac{E\left[C\left(G^{\prime}\right)\right]-E[C(G)]}{E[C(G)]} \\
(\%)\end{array}$ & $p$ \\
\hline $0.5-4 \mathrm{~Hz}$ & 0.001218 & 0.001226 & 0.66 & 0.709 \\
$4-8 \mathrm{~Hz}$ & $3.358 \times 10^{-5}$ & $4.428 \times 10^{-5}$ & 32 & 0.041 \\
$8-10 \mathrm{~Hz}$ & 0.01271 & 0.006480 & -49 & 0.383 \\
$10-13 \mathrm{~Hz}$ & 0.005229 & 0.004266 & -18 & 0.353 \\
$13-30 \mathrm{~Hz}$ & $1.6891 \times 10^{-5}$ & $1.9424 \times 10^{-5}$ & 15 & 0.005 \\
\hline \hline
\end{tabular}

\section{Weighted clustering coefficient}

As proposed in [28], the weighted clustering coefficient of node $i$ is

$$
c_{i}=\frac{1}{d_{i}\left(d_{i}-1\right)} \sum_{\substack{j=1 \\ j \neq i}}^{N} \sum_{\substack{k=1 \\ k \neq j, i}}^{N}\left(w_{i j} w_{i k} w_{j k}\right)^{1 / 3},
$$

where $d_{i}$ is the degree of node $i$. The contribution of each triangle to the clustering coefficient $c_{i}$ depends on all constituent link weights. Thus, a triangle in which each link has a link weight of 1 contributes unity to the sum, while a triangle having one link with a negligible weight will have a negligible contribution to the clustering coefficient. The weighted clustering coefficient $C=\frac{1}{N} \sum_{i=1}^{N} c_{i}$ of a network is the average over all the nodes. Given the set of link weights in a network, a large weighted clustering coefficient implies that links within a triangle tend to possess similar link weights or, equivalently, a positive link weight correlation within triangles. Since the link weight distributions before and after tumor resection are similar, the change in link weight correlation within triangles of a network after neurosurgery can be revealed via the changes in the weighted clustering coefficient. As shown in Table IV, the average weighted clustering coefficient decreases greatly $\left\{E\left[C\left(G^{\prime}\right)\right]\right.$ $-E[C(G)]\} / E[C(G)]=-49 \%$ in $8-10 \mathrm{~Hz}$, which is however statistically not significant because $p=0.383>0.05$. The increase in the weighted clustering coefficient in the $13-30 \mathrm{~Hz}$ band is statistically significant. In previous studies, decreased clustering coefficients in this frequency band have been found in brain tumor patients when compared to healthy participants $[8,9]$. The current results suggest—as was the case with theta band connectivity findings [10] — that a normalization of beta band clustering may occur after the removal of the tumor in these patients.

Comparing Tables III and IV, we find that $\left\{E\left[C\left(G^{\prime}\right)\right]\right.$ $-E[C(G)]\}\left\{E\left[\Sigma_{r}\left(G^{\prime}\right)\right]-E\left[\Sigma_{r}(G)\right]\right\}<0$ holds in each frequency band except for in $0.5-4 \mathrm{~Hz}$ where $\left(\left\{E\left[C\left(G^{\prime}\right)\right]\right.\right.$ $\left.-E[C(G)]\}\left\{E\left[\Sigma_{r}\left(G^{\prime}\right)\right]-E\left[\Sigma_{r}(G)\right]\right\}\right) / E[C(G)] E\left[\Sigma_{r}\right] \rightarrow 0$. After neurosurgery, the average weighted clustering coefficient increases (decreases) when the link weight correlation around a node increases (decreases), which corresponds to the decrease (increase) in $\Sigma_{r}$. If link weights around each node are positively correlated, any two links in a triangle are connected to a same node, thus tending to have similar link weight. As a result, all links within a triangle possess similar link weights. The link weight correlations around nodes and within triangles are positively correlated.

\section{Maximum spanning tree}

The maximum spanning tree (MaST) of a network is a tree spanning over all the nodes of a network, while the total weight of these $N-1$ links is maximal. Similar to Kruskal's minimum spanning tree algorithm $[29,30]$, a MaST starts as empty and is constructed as follows: (a) sort all $L$ links in decreasing order of their link weights and (b) proceed from the highest link weight to the smallest: a link belongs to the MaST if this link addition allows the MaST to span more nodes and no loop is generated. The process of constructing the MaST ends when the MaST spans all the nodes in the network

We have examined the link weight correlation surrounding nodes and within triangles in Secs. III B and III C. Here, via the MaST, we investigate whether high link weights are distributed all over the network or are isolated in a subgraph of the network. Recall that a link weight has rank $r$ if it is the $r$ th highest in a network. We use $r_{\min }(\mathrm{MaST})$ to denote the rank of the minimum link weight in a MaST. In the construction of a MaST, the last link joining the MaST has the smallest link weight $w_{\min }(\mathrm{MaST})$ and the largest rank $r_{\min }(\mathrm{MaST})$ compared to other links in the MaST. The rank dominance $\Delta(G)$ of the MaST in a graph $G$ can be defined by

$$
\Delta(G)=\frac{L-r_{\min }(\mathrm{MaST})}{L-N-1},
$$

where $L$ is the number of links in the network $G$. Clearly, $0 \leq \Delta \leq 1$ and when $r_{\min }(\mathrm{MaST})=N-1$, then $\Delta=1$ : the MaST is composed of the $N-1$ links with the highest link weight in the network. However, if a node is connected with the rest of the network by only one link, whose link weight is the minimum of the network, then this link belongs to the MaST, and $r_{\text {min }}(\mathrm{MaST})=L$ such that $\Delta=0$. The percentage of the network's links whose weight is smaller than the minimum link weight in the MaST approximates $\Delta$, i.e., $\operatorname{Pr}\left[w \leq w_{\text {min }}(\mathrm{MaST})\right] \simeq \Delta$. When high link weights appear only in a subgraph, $r_{\min }(\mathrm{MaST})$ would be large and the rank dominance $\Delta$ would be small. Therefore, a large $\Delta$ value indicates that high link weights are distributed all over the network such that all nodes can be reached only via high link weights. On the other hand, a small $\Delta$ value implies that high link weights appear only in a subgraph.

The average rank dominance of networks measured before and after neurosurgery over different frequency bands is given in Table V. Most brain networks possess a high $\Delta(G)$ value. Hence, relatively high link weights are at least not isolated in a subgraph, but are evenly spread over the entire brain. Table $\mathrm{V}$ shows that statistically significant changes $(p=0.00<0.05)$ occur in band $13-30 \mathrm{~Hz}$ : the average rank dominance decreases by $\left\{E\left[\Delta\left(G^{\prime}\right)\right]-E[\Delta(G)]\right\} / E[\Delta(G)]$ $=-1.7 \%$. This change suggests that after neurosurgery, higher link weights are increasingly present in only a subgraph of the network. Why this change occurs is not yet understood. Speculatively, this rank dominance could be re- 
TABLE V. The rank dominance $\Delta$ of networks measured before and after neurosurgery and its statistical tests.

\begin{tabular}{ccccc}
\hline \hline & $\begin{array}{c}E[\Delta(G)] \text { before } \\
\text { surgery }\end{array}$ & $\begin{array}{c}E\left[\Delta\left(G^{\prime}\right)\right] \text { after } \\
\text { surgery }\end{array}$ & $\begin{array}{c}\frac{E\left[\Delta\left(G^{\prime}\right)\right]-E[\Delta(G)]}{E[\Delta(G)]} \\
(\%)\end{array}$ & $p$ \\
\hline $0.5-4 \mathrm{~Hz}$ & 0.907 & 0.906 & -0.11 & 0.527 \\
$4-8 \mathrm{~Hz}$ & 0.945 & 0.938 & -0.74 & 0.068 \\
$8-10 \mathrm{~Hz}$ & 0.896 & 0.914 & 2.0 & 0.739 \\
$10-13 \mathrm{~Hz}$ & 0.927 & 0.928 & 0.11 & 0.829 \\
$13-30 \mathrm{~Hz}$ & 0.962 & 0.946 & -1.7 & 0.000 \\
\hline \hline
\end{tabular}

lated to the degree of subspecialization in the network, which may indicate that subgrouping in modules is enhanced after neurosurgery. One previous study shows that patients with epilepsy have altered modularity in the brain: although the number of modules was comparable to healthy participants, the role of the modules was changed in patients [31].

Furthermore, the rank dominance $\Delta(G)$ of a graph $G$ is positively correlated with its link weight correlation $\Sigma_{r}(G)$ of links surrounding a node. This is verified in functional brain networks. First, comparison of Table III with Table $\mathrm{V}$ gives $\left\{E\left[\Delta\left(G^{\prime}\right)\right]-E[\Delta(G)]\right\}\left\{E\left[\Sigma_{r}\left(G^{\prime}\right)\right]-E\left[\Sigma_{r}(G)\right]\right\}>0$. Second, the positive correlation between $\Sigma_{r}$ and $\Delta$ is illustrated by the 120 functional brain networks measured both before and after neurosurgery in each frequency band. The scatter plots are not shown here, because Figs. 6 and 7 show that both $\Sigma_{r}$ and $\Delta$ are negatively correlated with the spectral radius $\lambda_{1}$ of the graph. When link weights incident to a node are strongly positively correlated, a node that is surrounded by small link weights will be reached or spanned by the MaST via a small link weight. In this case, $r_{\min }(\mathrm{MaST})$ is large and $\Delta$ is small. Therefore, a strong positive link weight correlation around a node leads to a MaST including low link weight or a small $\Delta$. In other words, a strong positive link weight correlation contributes to the clustering of high link weights in a subgraph. This agrees with Ramasco and Gonçalves' observation [14] that a stronger positive link weight correlation around nodes implies a superhighway with larger size and higher weight in total, where a superhighway is the incipient percolation cluster (IIC), a subgraph of the MaST that is used by transport [32] more often. A strong positive link weight correlation around a node is thus claimed to improve the global transport. In the brain, this type of positive link weight correlation, or assortativity, may be beneficial to spread information throughout all parts of the brain in a fast and efficient manner. It seems that, even in brain tumor patients, this characteristic of the brain's network is preserved.

\section{E. Weighted spectrum}

A weighted network $G$ consisting of $N$ nodes and $L$ links can be described by the weighted adjacency matrix $A$, a $N \times N$ 0-1 matrix, where the element $a_{i j}=w_{i j}$ if there is a link between nodes $i$ and $j$; else $a_{i j}=0$. Since $A$ is a symmetric matrix, all eigenvalues are real and $A$ possesses an eigenvalue decomposition [33], Art. 9, p. 443,

$$
A=X \Lambda X^{T},
$$

where $X=\left[\begin{array}{llll}x_{1} & x_{2} & \ldots & x_{N}\end{array}\right]$ is an orthogonal matrix (such that $X^{T} X=I$ ) with as columns the real and normalized eigenvectors $x_{1}, x_{2}, \ldots, x_{N}$ of $A$, corresponding to the eigenvalues $\lambda_{1} \geq \lambda_{2} \geq \cdots \geq \lambda_{N-1} \geq \lambda_{N}$ in descending order and the diagonal matrix $\Lambda=\operatorname{diag}\left(\lambda_{1}, \lambda_{2}, \ldots, \lambda_{N-1}, \lambda_{N}\right)$. The set of eigenvalues $\lambda_{1} \geq \lambda_{2} \geq \cdots \geq \lambda_{N-1} \geq \lambda_{N}$ is called the spectrum of a network $G$. The spectrum is conjectured [34] to be the unique fingerprint of a large network. Recall Wigner's Semicircle Law [35]

Theorem 1. If the link weights $a_{i j}=w_{i j}$ are independent and identically distributed with $\sigma^{2}=\operatorname{Var}\left[w_{i j}\right]$, thus uncorrelated, the probability function $f_{\lambda}\left(A_{N}\right)(x)$ of an eigenvalue $\lambda^{\left(A_{N}\right)}$ of the scaled matrix $A_{N}=\frac{A}{\sqrt{N}}$ tends for $N \rightarrow \infty$ to

$$
\lim _{N \rightarrow \infty} f_{\lambda^{(}\left(A_{N}\right)}(x)=\frac{1}{2 \pi \sigma^{2}} \sqrt{4 \sigma^{2}-x^{2}} 1_{|x| \leq 2 \sigma} .
$$

Hence, for sufficiently large $N$, the distribution of the eigenvalues of $\frac{A}{\sqrt{N}}$ does not depend anymore on the link weight distribution. We first illustrate the semicircle law by an example. We take one functional brain network whose spectrum, actually the probability density function of eigenvalues, is displayed in Fig. 4(a). The spectrum is far from a semicircle, because link weights are positively correlated around nodes and within triangles as illustrated in Secs. III B and III C. After all the link weights are randomly reshuffled, they are not correlated anymore. Figure 4(b) shows the spectrum of $10^{4}$ reshuffled (see Sec. III B) networks, which fol-

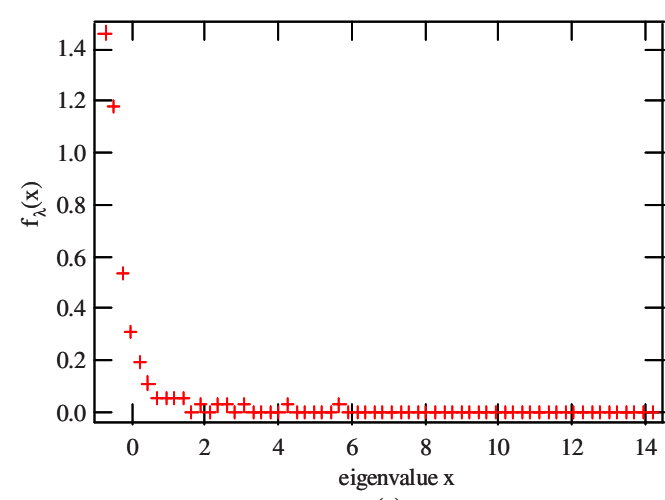

(a)

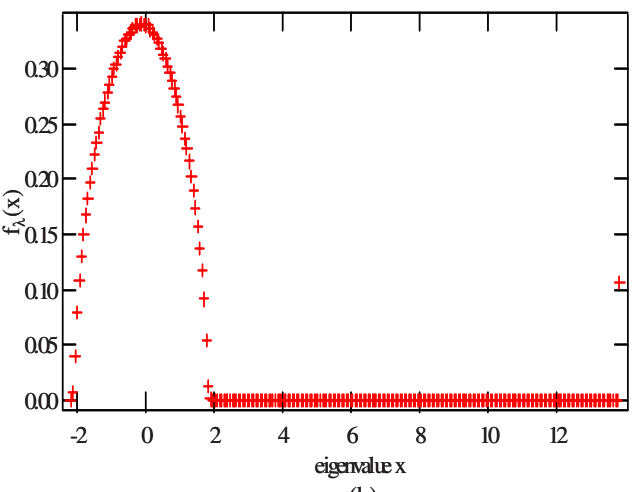

(b)

FIG. 4. (Color online) The spectra of one brain functional network and of the $10^{4}$ weight-reshuffled networks. 

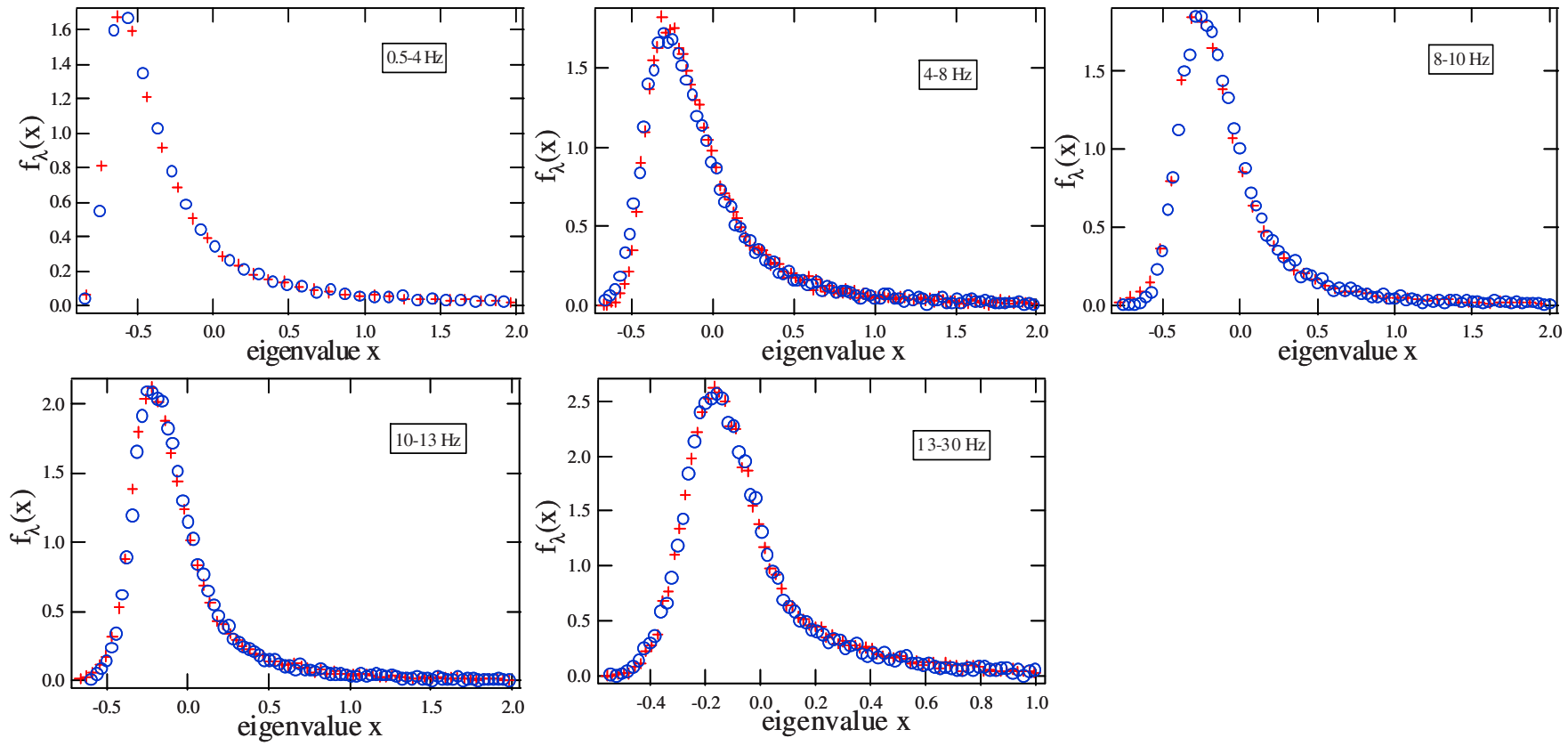

FIG. 5. (Color online) The spectra (in the range around zero) of the brain function networks measured via MEG before (red crosses) and after (blue circles) tumor resection.

lows nicely the Wigner semicircle law. The spectrum of the functional brain networks measured before and after tumor resection is depicted in Fig. 5. The spectrum over $0.5-4 \mathrm{~Hz}$ is farther from the semicircle centered at the origin compared to all the other frequency bands, implying that link weights are strongly correlated over $0.5-4 \mathrm{~Hz}$. This observation is consistent with what we have discovered in Table III: the average link weight correlation $E\left[\Sigma_{r}\right]$ in $0.5-4 \mathrm{~Hz}$ is smaller than in the other frequency bands.

Actually, the largest eigenvalue may well measure the link weight correlation of a network and characterize the dynamic processes on the network. The largest eigenvalue $\lambda_{1}$, also called the spectral radius, follows

$$
\lambda_{1}=\sup _{x \neq 0} \frac{x^{T} A x}{x^{T} x}
$$

and the maximum is attained if and only if $x$ is the eigenvector of $A$ belonging to $\lambda_{1}$. For any other vector $y \neq x$, it holds that $\lambda_{1} \geq x^{T} A x / x^{T} x$. By choosing the vector $y=u$ $=(1,1, \ldots, 1)$, we have

$$
\lambda_{1} \geq \frac{1}{N} \sum_{i=1}^{N} \sum_{j=1}^{N} w_{i j}=\frac{1}{N} \sum_{i=1}^{N} s_{i}=E_{G}[S]
$$

where $s_{i}$ is the node strength of node $i$ and $E_{G}[S]$ is the average node strength of the graph $G$. When $y$ is any 0-1 vector such as $y=(1,0,1, \ldots, 0), y^{T} A y / y^{T} y$ gives the average node strength $E_{G_{s}}[S]$ of a subgraph composed of the set of nodes corresponding to the 1's in $y$ and links in between the set of nodes. Therefore, the spectral radius is lower bounded by

$$
\lambda_{1} \geq \max \left\{E_{G_{S}}[S], E_{G}[S]\right\} .
$$

When link weights are highly correlated such that a large subgraph composed mostly of high link weights exists, the large average node strength of this subgraph tends to render a large spectral radius. In other words, the largest eigenvalue may capture the link weight correlation, in the sense that large link weights cluster in a subgraph.

Intuitively, the link weight correlation around nodes or within triangles contributes to the existence of such a subgraph with large average node strength, thus leading to a higher spectral radius. This conjecture is supported by Ramasco and Gonçalves' observation [14] as mentioned in Sec. III D. Rigorously, we show in [36], for all integers $k \geq 1$, that

$$
\lambda_{1} \geq\left(\frac{u^{T} A^{2 k} u}{u^{T} u}\right)^{1 / 2 k} \geq\left(\frac{u^{T} A^{k} u}{u^{T} u}\right)^{1 / k},
$$

from which $\lim _{k \rightarrow \infty}\left(u^{T} A^{k} u / N\right)^{1 / k}=\lambda_{1}$. Thus, $\lambda_{1} \geq u^{T} A u / u^{T} u$ leads to Eq. (4) and

$$
\lambda_{1} \geq \sqrt{\frac{u^{T} A^{2} u}{u^{T} u}}=\sqrt{\frac{1}{N} \sum_{k=1}^{N} s_{k}^{2}}=\sqrt{\operatorname{Var}[S]+(E[S])^{2}} .
$$

Functional brain networks measured before and after neurosurgery possess a similar link weight distribution, and thus a similar average node strength $E[S]$. A stronger link weight correlation around a node $\Sigma_{r}$ contributes to a large variance $\operatorname{Var}[S]$ of node strength. This is observed in functional brain networks (see the pdf of the node strength in Fig. 2 and $E\left[\Sigma_{r}\right]$ in Table III). Hence, a strong link weight correlation around a node (a small $\Sigma_{r}$ ) implies a large spectral radius $\lambda_{1}$. Figure 6 shows the negative correlation between $\Sigma_{r}$ and $\lambda_{1}$ in the 120 functional brain networks in each frequency band. Finally, both a large spectral radius $\lambda_{1}$ and a small rank 

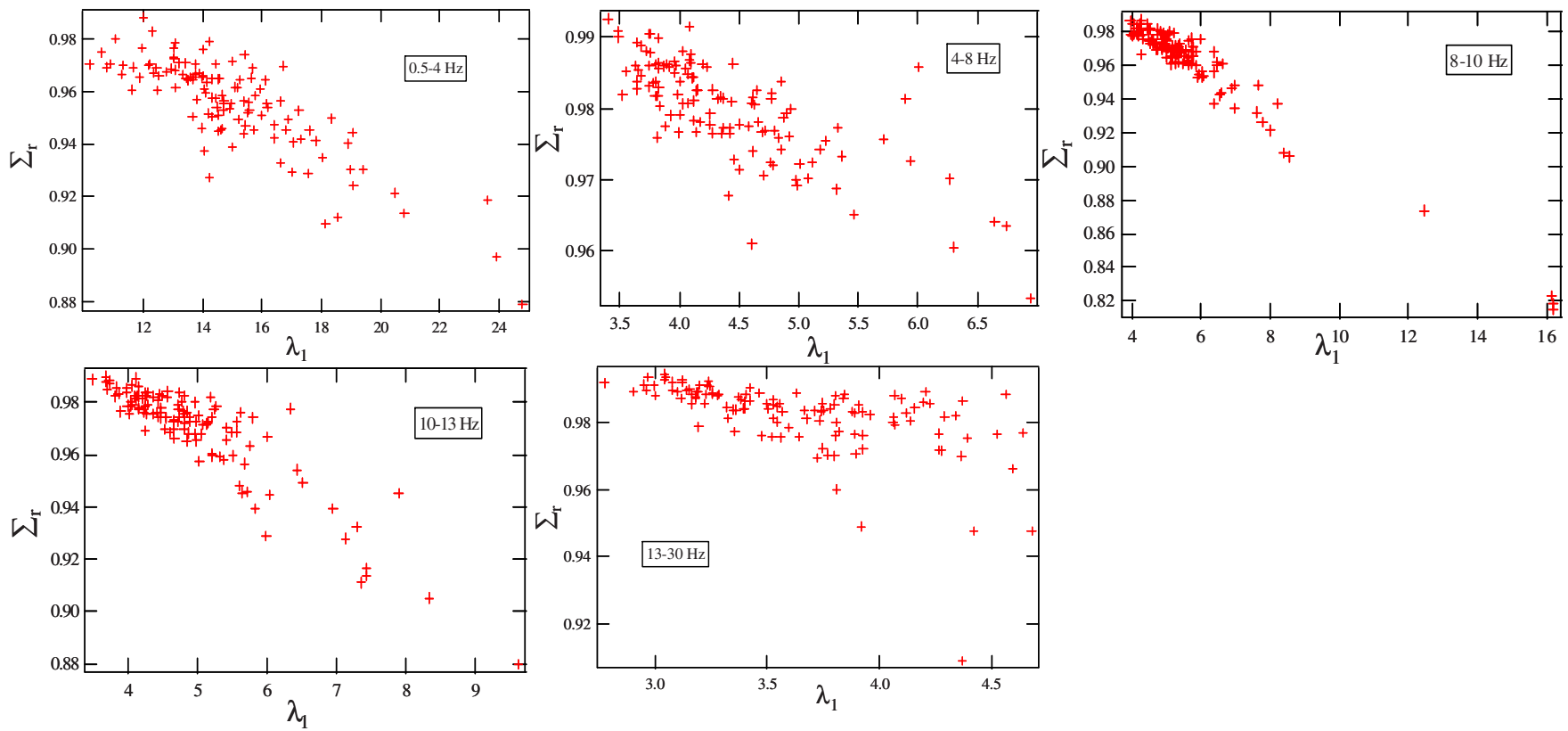

FIG. 6. (Color online) The scatter plot of link weight correlation around a node $\Sigma_{r}$ versus the largest eigenvalue $\lambda_{1}$ of each of the networks measured before and after tumor resection.

dominance $\Delta$ imply strong clustering of high link weights in a subgraph. Figure 7 demonstrates the negative correlation between $\Delta$ and the largest eigenvalue $\lambda_{1}$.

The changes in $\lambda_{1}$ introduced by neurosurgery are given in Table VI. The largest eigenvalue increases statistically significantly in the $13-30 \mathrm{~Hz}$ frequency band, indicating again that assortative beta band local clustering within subregions of the brain tends to increase after tumor removal in these patients. The susceptible-infected-susceptible virus spreading [37] and the Kuramoto type of synchronization process of coupled oscillators [38] have been characterized on a given but general network topology. Both dynamic and nonlinear processes feature a phase transition that specifies the onset of a remaining fraction of infected nodes and of locked oscillators, respectively. The more curious aspect is that each of the phase transitions in these different processes occurs at an effective spreading rate $\tau_{c}$ and coupling strength $g_{c}$, respectively, that is proportional to $1 / \lambda_{1}$. After surgery, the decrease in synchronization and/or spreading threshold in the 13-30 Hz frequency band implies the improvement of infor-
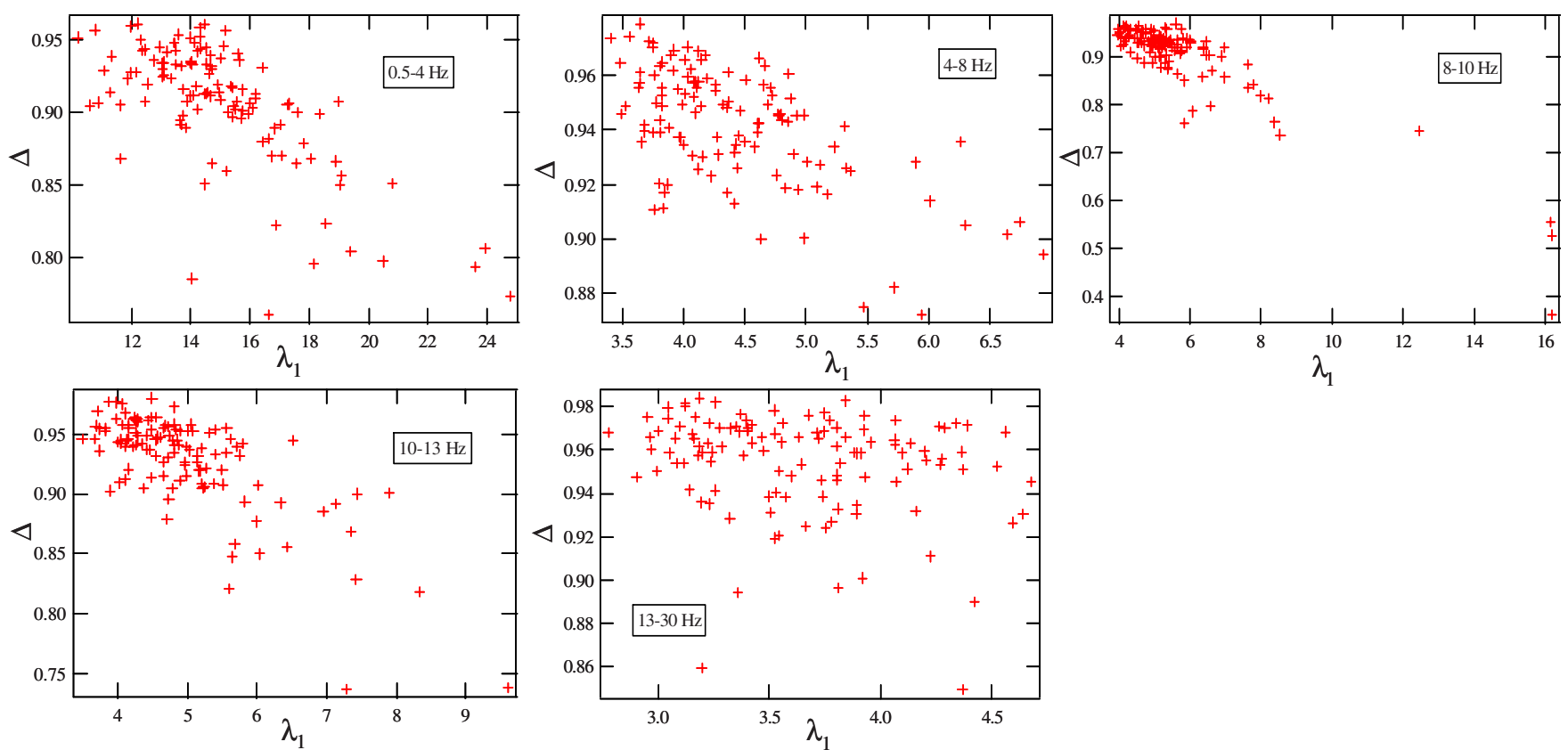

FIG. 7. (Color online) The scatter plot of rank dominance $R D$ versus the largest eigenvalue $\lambda_{1}$ of each of the networks measured before and after tumor resection. 
TABLE VI. The largest eigenvalue $\lambda_{1}$ of networks measured before and after neurosurgery and its statistical tests.

\begin{tabular}{ccccc}
\hline \hline & $\begin{array}{c}E\left[\lambda_{1}(G)\right] \\
\text { before } \\
\text { surgery }\end{array}$ & $\begin{array}{c}E\left[\lambda_{1}\left(G^{\prime}\right)\right] \\
\text { after } \\
\text { surgery }\end{array}$ & $\begin{array}{c}\frac{E\left[\lambda_{1}\left(G^{\prime}\right)\right]-E\left[\lambda_{1}(G)\right]}{E\left[\lambda_{1}\left(G^{\prime}\right)\right]} \\
(\%)\end{array}$ & $p$ \\
\hline $0.5-4 \mathrm{~Hz}$ & 14.8599 & 15.0620 & 1.36 & 0.551 \\
$4-8 \mathrm{~Hz}$ & 4.2700 & 4.5650 & 6.9 & 0.061 \\
$8-10 \mathrm{~Hz}$ & 5.8706 & 5.4793 & -6.7 & 0.561 \\
$10-13 \mathrm{~Hz}$ & 5.0542 & 4.8587 & -3.9 & 0.419 \\
$13-30 \mathrm{~Hz}$ & 3.5504 & 3.7193 & 4.8 & 0.002 \\
\hline \hline
\end{tabular}

mation processing capability. The positive correlations among link weight correlation around a node, the clustering of large link weights in a subgraph, and the spectral radius explain why $\left\{E\left[\lambda_{1}\left(G^{\prime}\right)\right]-E\left[\lambda_{1}(G)\right]\right\}\left\{E\left[\Sigma_{r}\left(G^{\prime}\right)\right]-E\left[\Sigma_{r}(G)\right]\right\}$ $<0$ and $\left\{E\left[\lambda_{1}\left(G^{\prime}\right)\right]-E\left[\lambda_{1}(G)\right]\right\}\left\{E\left[\Delta\left(G^{\prime}\right)\right]-E[\Delta(G)]\right\}<0$ hold in each frequency band.

In summary, statistically significant changes have been observed in 13-30 Hz characterized by the increase in link weight correlation around a node, within triangles and in a subgraph, and by the increase in spectral radius, implying that traffic transportation has been improved both locally and globally. Although no statistically significant correlations were found between these changes in network topology and functional outcome in terms of epilepsy after surgery, these findings could point toward a normalization of brain functioning after the brain tumor is removed from these patients.

\section{RELATION BETWEEN WEIGHTED AND UNWEIGHTED NETWORK ANALYSES}

A weighted network is usually transformed into an unweighted network by sampling links which are "important" according to their link weights due to the simplicity of an unweighted network analysis. In functional brain networks, a high link weight represents high strength of the functional connectivity between two parts of the brain. Usually, an unweighted network is mapped as the union of links whose link weight is above a threshold $x$ or, equivalently, as the union of a number $L$ of links with the highest link weight in the original weighted network. When we choose a different value of $L$, a different view of the original weighted network is captured in the unweighted network. A representative number of links $L$ is difficult to obtain. In this sense, the analysis on the original weighted network is more reliable and accurate, although more complex [39].

In this section, we examine the relation between the weighted and unweighted analyses. We transform each weighted brain network into an unweighted network by selecting the $L=E[D] N / 2$ links with the highest link weight. Recall in Sec. III D that the MaST includes the high link weights that are necessary to span all the nodes of $G(N)$. Let $G_{s}(N)$ denote any subgraph that spans over all the nodes of the network $G(N)$. The minimum link weight in $G_{s}(N)$ is always smaller or equal to the smallest link weight in the $\operatorname{MaST}[40]$, i.e., $w_{\min }\left(G_{s}(N)\right) \leq w_{\min }(\operatorname{MaST})$. The rank of $w_{\text {min }}\left(G_{s}(N)\right)$ is then larger than that of $w_{\text {min }}(\mathrm{MaST})$. Therefore, a subgraph should contain at least $L \geq r_{\min }(\mathrm{MaST})$ links such that all nodes are connected. Approximately, $L$ $\geq\left(\begin{array}{c}N \\ 2\end{array}\right)(1-\Delta)$. Table $\mathrm{V}$ indicates that the unweighted network has to include at least $10 \%$ links, so that it connects all the nodes in one component. In this section, we examine, as an example, the case when the average degree $E[D]=30$ as well as $E[D]=50$ only if necessary [41]. We deduce from the weighted analysis the most studied topological characteristics of the corresponding unweighted networks: standard deviation of the degree, the degree correlation of nodes connected by a link, and the clustering coefficient of a network.

\section{A. Standard deviation of degree}

The degree distribution of the unweighted brain function networks, as illustrated in Fig. 8, approximately follows a Gaussian distribution both before and after neurosurgery. A Gaussian distribution $(1 / \sigma \sqrt{2 \pi}) \exp \left[-(x-\mu)^{2} / 2 \sigma^{2}\right]$ can be characterized by the average $\mu$ and the standard deviation $\sigma$. Each unweighted brain network has average degree $E[D]$ $=30$, as the way it is constructed. When link weights of a node are positively correlated, high (low) link weights are likely connected to a same node, which contributes to a high (low) degree node in the corresponding unweighted network. In this case, the variance of degree in the unweighted network is expected to be large. We claim that a strong positive link weight correlation around a node implies high-degree variance in the corresponding unweighted network.

The average standard deviation $E[\sigma[D]]$ of the unweighted functional brain networks measured before and after neurosurgery is presented in Table VII. The degree variance increases (decreases) after tumor resection if link weight correlation surrounding a node increases (decreases): $\left\{E\left[\Sigma_{r}\left(G^{\prime}\right)\right]-E\left[\Sigma_{r}(G)\right]\right\}\left\{E\left[\sigma\left[D\left(G^{\prime}\right)\right]\right]-E[\sigma[D(G)]]\right\}<0$ (see Tables III and VII).

\section{B. Degree correlation}

Assortativity refers to a preference for nodes to attach to others that are similar in degree, i.e., a highly connected node tends to be connected with other high-degree nodes. On the other hand, a network is disassortative if high-degree nodes tend to attach to low-degree nodes. The assortativity and disassortativity describe the degree correlation of nodes connected by a link, which is also referred to as the mixing pattern of a network. If links around node are positively correlated in a weighted network, the link weights around node $i$ and around node $j$ tend to possess a similar link weight as $w_{i j}$. Nodes $i$ and $j$ are likely to have a similar degree in the corresponding unweighted network. Hence, a strong positive link weight correlation around a node may imply assortativity in the unweighted network.

The degree correlation [42] can be measured by the average degree of the neighbors of degree $k$ nodes, $E\left[D_{\mathcal{N}(i)} \mid d_{i}\right.$ $=k]$, where $\mathcal{N}(i)$ are the direct neighbors of node $i$. If there is no degree correlation of neighboring nodes, $E\left[D_{\mathcal{N}(i)} \mid d_{i}=k\right]$ is independent of $k$. When $E\left[D_{\mathcal{N}(i)} \mid d_{i}=k\right]$ is an increasing (decreasing) function of $k$, nodes with high degree tend to con- 

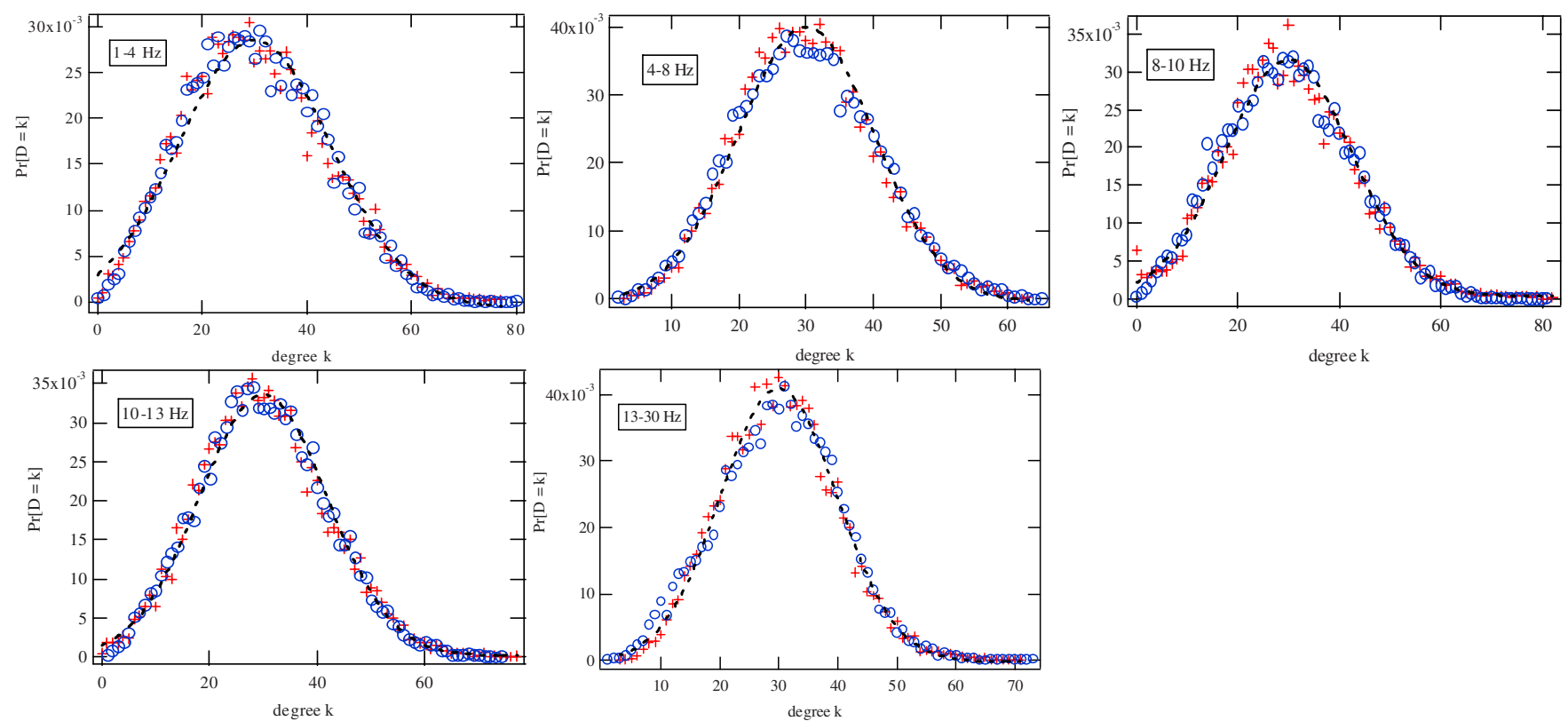

FIG. 8. (Color online) The degree distribution of the unweighted brain functional networks before (red crosses) and after surgery (blue circles) as well as the Gaussian curve fitting (black dotted line).

nect to high (low) degree nodes, and the network is classified as (dis)assortative.

As shown in Fig. 9, the unweighted networks follow a similar positive degree correlation before and after neurosurgery. The positive degree correlation is coherent with the positive link weight correlation of links connected to a same node, as shown in Sec. III B. However, the difference in degree correlations before and after neurosurgery is not evident.

\section{Clustering coefficient}

In an unweighted network, the clustering coefficient of a node $c_{i}^{u}$ characterizes the density of connections in the environment of a node $i$. The clustering coefficient is defined as the ratio of the number of links $y$ connecting the $d_{i}>1$ neighbors of $i$ over the total possible $d_{i}\left(d_{i}-1\right) / 2$; thus, $c_{i}^{u}$ $=2 y / d_{i}\left(d_{i}-1\right)$. The average clustering coefficient $C^{u}(G)$ of a network $G$ is the average clustering coefficient of nodes whose degree is larger than 1 . When the weighted clustering coefficient is large, links with large weight tend to appear in

TABLE VII. The standard deviation $\sqrt{\operatorname{var}[D]}$ of the unweighted brain functional networks measured before and after neurosurgery.

\begin{tabular}{cccc}
\hline \hline & $\begin{array}{c}E[\sigma[D(G)]] \\
\text { before } \\
\text { surgery }\end{array}$ & $\begin{array}{c}E\left[\sigma\left[D\left(G^{\prime}\right)\right]\right] \\
\text { after } \\
\text { surgery }\end{array}$ & $\begin{array}{c}\frac{E\left[\sigma\left[D\left(G^{\prime}\right)\right]\right]-E[\sigma[D(G)]}{E[\sigma[D(G)]]} \\
(\%)\end{array}$ \\
\hline $0.5-4 \mathrm{~Hz}$ & 13.228 & 12.918 & -2.3 \\
$4-8 \mathrm{~Hz}$ & 9.756 & 10.254 & 5.1 \\
$8-10 \mathrm{~Hz}$ & 12.643 & 12.336 & -2.4 \\
$10-13 \mathrm{~Hz}$ & 11.796 & 11.644 & -1.3 \\
$13-30 \mathrm{~Hz}$ & 9.597 & 10.221 & 6.5 \\
\hline \hline
\end{tabular}

a triangle. Such triangles possessing three high link weights are likely to remain in the corresponding unweighted network, which contribute to a large clustering coefficient of the unweighted network. Therefore, a strong link weight correlation within triangles or a large weighted clustering coefficient implies a high clustering coefficient in the unweighted network. In Table VIII, we present the average clustering coefficient $E\left[C^{u}\right]$ of the unweighted functional brain networks measured before and after neurosurgery. The average clustering coefficient increases slightly after tumor resection over each frequency band when $E[D]=30$. The decreases over $8-10$ and $10-13 \mathrm{~Hz}$ implied by weighted clustering coefficient in Table IV are not observed. When we map the unweighted network with more links, $E[D]=50$, the decrease in clustering coefficient over 10-13 can be recognized, as given in Table VIII.

The evident features in the unweighted networks deduced from the weighted analysis, such as assortativity and the change in degree variance after surgery, have been observed in the case $E[D]=30$. However, the unweighted network captures the information of the original weighted network only partially. Some changes in weighted network features like the weighted clustering coefficient cannot be revealed in unweighted networks mapped with only $E[D]=30$. The weighted network analysis turns out to be crucial, especially to exploring the changes in network properties. This motivates the weighted analysis as a potentially standard approach in future neuroscience studies.

\section{CONCLUSION}

The functional brain networks of a group of patients with brain tumors have been measured before and after tumor resection. In this work, we perform a weighted network analysis to understand the effects of neurosurgery on features 

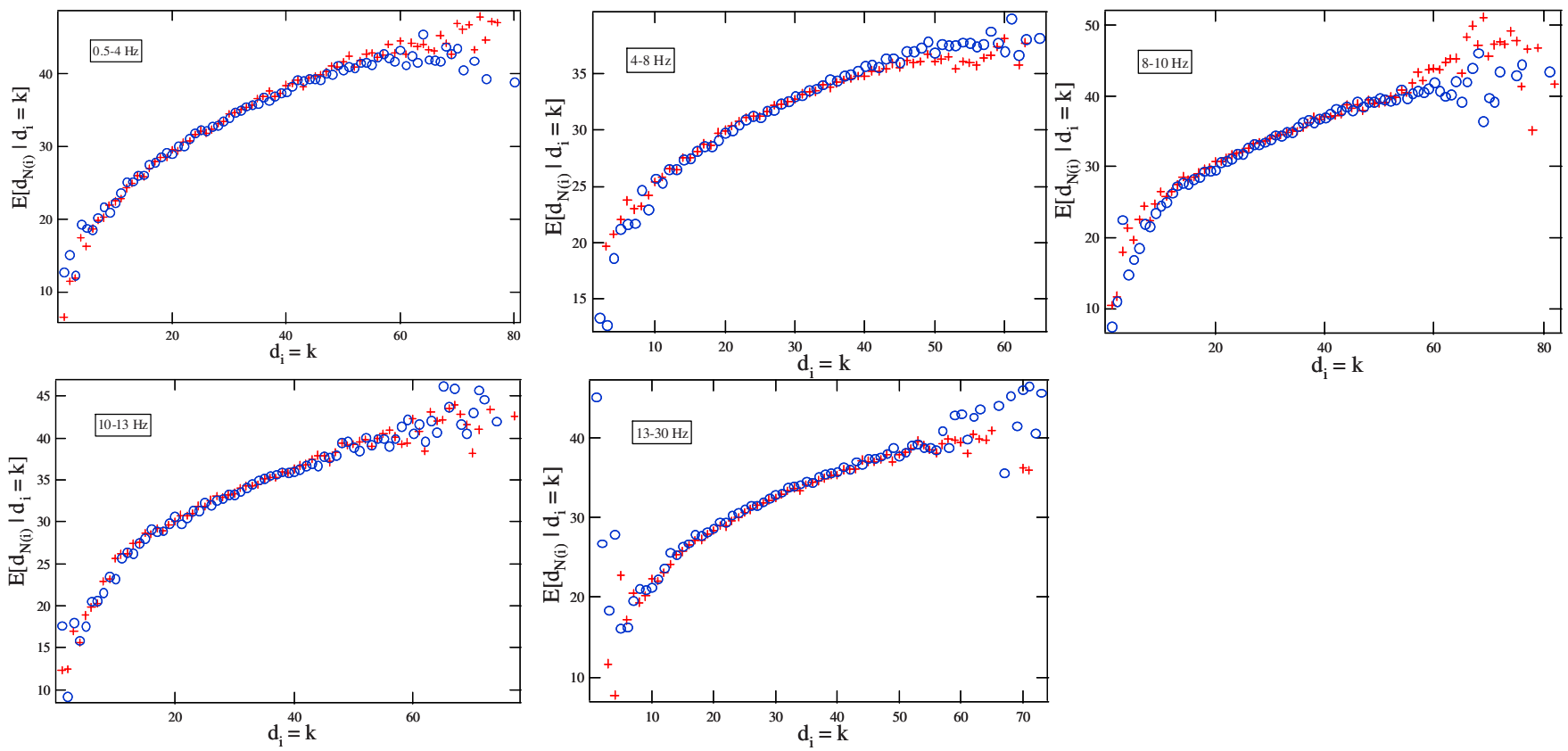

FIG. 9. (Color online) The average degree $E\left[D_{\mathcal{N}(i)} \mid d_{i}=k\right]$ of the neighbors of nodes with a given degree $k$, in the unweighted networks measured before (red crosses) and after surgery (blue circles).

of functional brain networks. We introduce innovative measures to examine link weight correlations around a node, within a triangle and in a subgraph. Furthermore, by investigating the spectral radius, we set up the relation between the link weight correlation of a network and dynamic processes such as synchronization on the network.

Our statistical tests show that neurosurgery induces statistically significant changes mainly in the $13-30 \mathrm{~Hz}$ frequency band: the link weight correlation around nodes, within triangles as well as in a subgraph, and the spectral radius all increase in this frequency band. A strong positive correlation of link weights surrounding a node contributes to (a) a strong link weight correlation within triangles and to (b) the clustering of high link weights in a large subgraph. The former (a) associates with high local efficiency of information transfer and robustness [43]. The latter (b) introduces a large incipient percolation cluster (IIC) in both size and weight, which enhances the global transport capability [14]. The increase in the spectral radius, or the decrease in synchronization and/or spreading threshold, again, suggests the improve- ment of information dissemination. Previous studies in brain tumor patients have shown that local clustering is decreased in the beta band when compared to healthy controls $[8,9]$. The current results-although investigated differently with respect to methodology-indicate that this disturbance in network topology that occurs in brain tumor patients may be normalized after the removal of the tumor. However, in the absence of a healthy control population in this study, this remains to be elucidated. Additionally, whether the decrease in synchronization threshold indicates, e.g., the increase in seizure frequency or cognitive status remains an open question. In $8-10 \mathrm{~Hz}$, evident decreases in these link weight correlations and the spectral radius have been observed in a small number of patients, although these changes are statistically not significant due to great variance of SL in this frequency band.

Finally, weighted networks are traditionally transformed into unweighted networks by mapping $L$ links with the highest link weight and are studied by unweighted network analysis afterward. We have investigated the relation be-

TABLE VIII. The average clustering coefficient of the unweighted brain functional networks measured before $E\left[C^{u}(G)\right]$ and after $E\left[C^{u}\left(G^{\prime}\right)\right]$ neurosurgery.

\begin{tabular}{|c|c|c|c|c|c|c|}
\hline & \multicolumn{3}{|c|}{$E[D]=30$} & \multicolumn{3}{|c|}{$E[D]=50$} \\
\hline & $E\left[C^{u}(G)\right]$ & $E\left[C^{u}\left(G^{\prime}\right)\right]$ & $\frac{E\left[C^{u}\left(G^{\prime}\right)\right]-E\left[C^{u}(G)\right]}{E\left[C^{u}\left(G^{\prime}\right)\right]}\left(\begin{array}{c}\%) \\
(\%)\end{array}\right.$ & $E\left[C^{u}(G)\right]$ & $E\left[C^{u}\left(G^{\prime}\right)\right]$ & 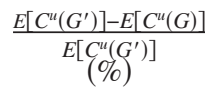 \\
\hline $0.5-4 \mathrm{~Hz}$ & 0.5017 & 0.5045 & 0.56 & 0.5533 & 0.5539 & 0.11 \\
\hline $4-8 \mathrm{~Hz}$ & 0.3909 & 0.4086 & 4.5 & 0.4484 & 0.4583 & 2.2 \\
\hline $8-10 \mathrm{~Hz}$ & 0.4313 & 0.4455 & 3.3 & 0.4920 & 0.4965 & 0.91 \\
\hline $10-13 \mathrm{~Hz}$ & 0.4257 & 0.4276 & 0.45 & 0.4809 & 0.4784 & -0.52 \\
\hline $13-30 \mathrm{~Hz}$ & 0.4308 & 0.4556 & 5.8 & 0.4507 & 0.4658 & 3.4 \\
\hline
\end{tabular}


tween the weighted and unweighted network analysis results and illustrate that the weighted analysis is more reliable and accurate than the unweighted network analysis, which always captures only partial information of the original weighted network.

\section{ACKNOWLEDGMENTS}

We thank J. C. Baayen and J. C. de Munck for collection of the data used in this study.
[1] M. E. J. Newman, SIAM Rev. 45, 167 (2003).

[2] L. da F. Costa, F. A. Rodrigues, G. Travieso, and P. R. Villas Boas, Adv. Phys. 56, 167, 2007.

[3] H. Wang, J. M. Hernandez, and P. Van Mieghem, Phys. Rev. E 77, 046105 (2008).

[4] D. J. Watts and S. H. Strogatz, Nature (London) 393, 440 (1998).

[5] J. C. Reijneveld, S. C. Ponten, H. W. Berendse, and C. J. Stam, Clin. Neurophysiol. 118, 2317 (2007).

[6] C. J. Stam and J. C. Reijneveld, Nonlinear Biomed. Phys. 1, 3 (2007).

[7] A. M. Aertsen, G. L. Gerstein, M. K. Habib, and G. Palm, J. Neurophysiol. 61, 900 (1989).

[8] F. Bartolomei, I. Bosma, M. Klein, J. C. Baayen, J. C. Reijneveld, T. J. Postma, J. J. Heimans, B. W. van Dijk, J. C. de Munck, A. de Jongh, K. S. Cover, and C. J. Stam, Clin. Neurophysiol. 117, 2039 (2006).

[9] I. Bosma, J. C. Reijneveld, M. Klein, L. Douw, B. W. van Dijk, J. J. Heimans, and C. J. Stam, Nonlinear Biomed. Phys. 3, 9 (2009).

[10] L. Douw, H. Baayen, I. Bosma, M. Klein, P. Vandertop, J. Heimans, K. Stam, J. de Munck, and J. Reijneveld, Exp. Neurol. 212, 285 (2008).

[11] A. Barrat, M. Barthélemy, R. Pastor-Satorras, and A. Vespignani, Proc. Natl. Acad. Sci. U.S.A. 101, 3747 (2004).

[12] J.-P. Onnela, J. Saramäki, J. Hyvonen, G. Szabó, M. Argollo de Menezes, K. Kaski, A.-L. Barabási, and J. Kertész, New J. Phys. 9, 179 (2007).

[13] M. Á. Serrano, M. Boguñá, and R. Pastor-Satorras, Phys. Rev. E 74, 055101(R) (2006).

[14] J. J. Ramasco and B. Gonçalves, Phys. Rev. E 76, 066106 (2007).

[15] L. Douw, J. C. Baayen, M. Klein, D. Velis, W. C. Alpherts, J. Bot, J. J. Heimans, J. C. Reijneveld, and C. J. Stam, Neuroimage 46, 584 (2009).

[16] C. J. Stam et al., Brain 132, 213 (2009).

[17] E. van Dellen et al., PLoS ONE 4, e8081 (2009).

[18] C. J. Stam and B. W. van Dijk, Physica D 163, 236 (2002).

[19] N. F. Rulkov, M. M. Sushchik, L. S. Tsimring, and H. D. Abarbanel, Phys. Rev. E 51, 980 (1995).

[20] D. S. Bassett, A. Meyer-Lindenberg, S. Achard, T. Duke, and E. Bullmore, Proc. Natl. Acad. Sci. U.S.A. 103, 19518 (2006).

[21] Y. He, Z. J. Chen, and A. C. Evans, Cereb. Cortex 17, 2407 (2007).

[22] G. Gong, Y. He, L. Concha, C. Lebel, D. W. Gross, A. C. Evans, and C. Beaulieu, Cereb. Cortex 19, 524 (2009).

[23] P. Van Mieghem and H. Wang, IEEE/ACM Trans. Netw. 17, 93 (2009).

[24] A weight-reshuffled graph can be obtained by collecting all the link weights in the original network and by randomly reassign- ing them to the links in the original network topology. Equivalently, half, e.g., upper right of the original adjacency matrix is randomly shuffled and then is mapped to the lower-left part of the adjaceny matrix, whose symmetry is reserved in this weight-reshuffling process.

[25] F. Wilcoxon, Biometrics 1, 80 (1945).

[26] With $N=150$, we are able to compare the link weight correlation around a node via $\left(s_{i}+s_{j}\right) / 2(N-1)$ versus $w_{i j}$ in networks measured before and after neurosurgery, because almost the same link weight distribution is followed.

[27] Instead of making scatter plots of all $\left[\left(s_{i}+s_{j}\right) / 2(N-1), w_{i j}\right]$ pairs which heavily overlap, we divide the link weight range $[0,1]$ into 100 bins, and over each bin we calculate the average $\left(s_{i}+s_{j}\right) / 2(N-1)$ corresponding to those $w_{i j}$ that belong to the same bin.

[28] J.-P. Onnela, J. Saramäki, J. Kertész, and K. Kaski, Phys. Rev. E 71, 065103(R) (2005).

[29] The maximum spanning tree in a weighted network $G$ with link weight $w_{i j} \in\left[w_{\min }, w_{\max }\right]$ is the same as the minimum spanning tree in $G$, while equipped with link weight $w_{i j}^{\prime}$ $=w_{\max }-w_{i j}$.

[30] Joseph B. Kruskal, Proc. Am. Math. Soc. 7, 48 (1956).

[31] M. Chavez, M. Valencia, V. Navarro, V. Latora, and J. Martinerie, Phys. Rev. Lett. 104, 118701 (2010).

[32] Transport is assumed to follow the shortest paths.

[33] P. Van Mieghem, Performance Analysis of Communications Networks and Systems (Cambridge University Press, Cambridge, England, 2006).

[34] E. R. van Dam and W. H. Haemers, Discrete Math. 309, 576 (2009).

[35] E. Wigner, Ann. Math. 67, 325 (1958).

[36] P. Van Mieghem, Graph Spectra for Complex Networks (Cambridge University Press, Cambridge, England) (to be published).

[37] P. Van Mieghem, J. Omic, and R. E. Kooij, IEEE/ACM Trans. Netw. 17, 1 (2009).

[38] J. G. Restrepo, E. Ott, and Brian R. Hunt, Phys. Rev. E 71, 036151 (2005).

[39] Weighted network analysis could be noisy if the weakest link weights may not represent real coupling, but statistical fluctuations in the coupling measure.

[40] The construction of a MaST shows that, if node $i$ is the last node to join the MaST by including link $(i, k), w_{i k}$ $=w_{\min }(\mathrm{MaST})$ and $w_{i k}$ must be the largest link weight among all links connected to node $i$, or else node $i$ can join the MaST by another link incident to it, but with higher link weight. Any other subgraph $G_{s}(N)$ spanning all nodes must contain at least one link connected to node $i$, whose weight is smaller or equal to $w_{i k}=w_{\min }(\mathrm{MaST})$ and which can be larger than $w_{\min }\left(G_{s}(N)\right)$. Hence, $w_{\min }\left(G_{s}(N)\right) \leq w_{\min }(\operatorname{MaST})$.

[41] Thus, $E[D] / N=20 \%$ of the original links with the highest link 
weights are included, resulting in a small number of networks with only few disconnected nodes. These disconnected nodes, although small in number, are all taken into account in the unweighted analysis, because such disconnectivity also reveals information about the weighted networks.
[42] R. Pastor-Satorras, A. Vazquez, and A. Vespignani, Phys. Rev. Lett. 87, 258701 (2001).

[43] E. Bullmore and O. Sporns, Nat. Rev. Neurosci. 10, 186 (2009). 